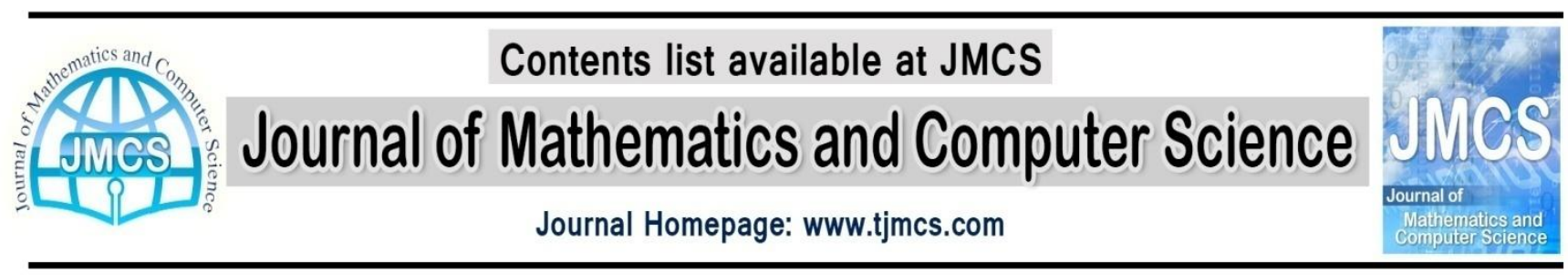

\title{
A method for calculating interval inverse matrix
}

\author{
Shohreh Abolmasoumi* \\ Department of Mathematics, Arak Branch, Islamic Azad University, Arak, Iran \\ sh.masoumi55@yahoo.com
}

Article history:

Received March 2014

Accepted April 2014

Available online May 2014

\begin{abstract}
This paper has presented a method for calculation the interval inverse matrix using the linear equation system. Linear equation systems have important in mathematics, engineering, statistics and other disciplines. These systems can vary in the interval are searcher who have been considered. Also whit this type of systems have been encountered in many applications. Different bases of interval calculation has been discussed in several articles $[2,5,6,7]$. The method used in calculation in inverse matrix real case, we can extend it to the interval mode. To calculate the interval inverse matrix we define many definition that are consist of interval one number, interval zero number and interval identity matrix. It is noted that the uniqueness of the calculated interval inverse matrix is not guaranteed.
\end{abstract}

Keywords: Interval linear systems, Interval number, Interval matrix, Interval identity matrix.

\section{1: Introduction}

This calculation takes place when the non-singular and square matrix and entries are real number. A computational approach to this type of matrices is linear equation system which first matrix is the matrix of coefficients and latter represent the unknown. This system is equated to the identity matrix. What kind of matrix should be taken as a right-hand side matrix? This should be to matrix which is supposed to mimic the identity matrix used in the real case, the so- called interval identity matrix. For this purpose we introduce several new definitions. The fuzzy linear equation system and fuzzy arithmetic and calculation fuzzy inverse matrix proposed by $[1,4]$.

The matrix identity includes the same two zero number, one number. Which are fundamental parts of interval identity matrix are introduced. The organization of the paper is follow. In section 2, the 
definitions related to interval number and interval matrix and arithmetic operation is described briefly. Section 3 includes some now definitions pertinent to calculating the inverse of an interval matrix and, 2 examples are given in section 4. Final section is a conclusion.

\section{2: Preliminary}

Definition (2-1): Really that the closed interval denoted by[a, b] is the set of real numbers given by

$$
[a, b]=\{x \in \mathcal{R}: a \leq x \leq b\}
$$

Although various other types of intervals (open, half-open ) appear throughout mathematics our work well center primarily on closed intervals. In this paper, the term interval will mean closed interval. We will adopt the convention of denoting intervals and their endpoints by capital letters. The left and right endpoints of an interval $X$ will be denoted by $\underline{X}$ and $\bar{X}$. Thus $X=[\underline{X}, \bar{X}]$.The intervals $X$ and $Y$ are said to be equal if they are the same sets.

$$
\mathrm{X}=\mathrm{Y} \text { if and only if } \underline{X}=\underline{X} \text { and } \bar{Y}=\bar{Y}
$$

The width of an interval $X$ is defined and denoted by $\quad \omega(X)=\bar{X}-\underline{X}$.

Definition (2-2):(Endpointformulas of the arithmetic operations)

Let us find and operational way to add interval. Since

$$
\begin{gathered}
x \in X \text { means that } \quad \underline{Y} \leq y \leq \bar{Y} \\
y \in Y \text { means that } \\
\underline{X} \leq x \leq \bar{X}
\end{gathered}
$$

We see by addition of inequalities that the numerical sum $\mathrm{x}+\mathrm{y} \in X+Y$ must

Satisfy

$$
\underline{X}+\underline{Y} \leq x+y \leq \bar{X}+\bar{Y}
$$

Hence

$$
\mathrm{X}+\mathrm{Y}=[\underline{X}+\underline{Y}, \bar{X}+\bar{Y}]
$$

And

$$
-Y=[-\bar{Y},-\underline{Y}]=\{y: y \epsilon Y\}
$$

For subtraction we add the inequalities

$$
\underline{X} \leq x \leq \bar{X} \text { and }-\underline{Y} \leq-y \leq-\bar{Y}
$$

To get

$$
\underline{X}-\bar{Y} \leq x-y \leq \bar{X}-\underline{Y}
$$

It that

$$
X-Y=[\underline{X}-\bar{Y}, \bar{X}-\underline{Y}]
$$

Defined product $X \times Y$ of two interval $X$ and $Y$, [8]

$$
X \times Y=[\text { mins, max } s], \text { where } s=\{\underline{X Y}, \underline{X} \bar{Y}, \bar{X} \underline{Y}, \overline{X Y}\}
$$

A two-dimensional interval vector

$$
\mathrm{X}=\left[X_{1}, X_{2}\right]=\left[\left(\underline{X_{1}}, \overline{X_{1}}\right),\left(\underline{X_{2}}, \overline{X_{2}}\right)\right]
$$

Can be represented as a rectangle in the $x_{1} x_{2}$-plans: it is the set of all point $\left(x_{1}, x_{2}\right)$ such that

$$
\underline{X_{1}} \leq \underline{x_{1}} \leq \overline{X_{1}} \quad, \quad \underline{X_{2}} \leq \underline{x_{2}} \leq \overline{X_{2}}
$$


Definition (2-3): Interval matrix, we mean a matrix whose elements are interval number. For example, we might have

$$
\mathrm{A}=\left[\begin{array}{ll}
A_{11} & A_{12} \\
A_{21} & A_{22}
\end{array}\right]=\left[\begin{array}{ll}
{[1,2]} & {[-1,1]} \\
{[0,4]} & {[-6,8]}
\end{array}\right]
$$

When the support of an interval number is given as $[X, \bar{X}]$, an interval number is said to be positive if $0 \leq \underline{X} \leq \bar{X}$.similarly, an interval number is said to be negative if $\underline{X} \leq \bar{X}<0$.Finally an interval number if $\underline{X} \leq 0 \leq \bar{X}$. An indicated in the top, the $i j$ th element $C_{i j}$ of the product $C_{i j}=A B$ of an $m$ by $p$ interval matrix $\mathrm{B}$ gives sharp bounds on the range

$$
C_{i j}=\left\{M_{i j}=\sum_{k=1}^{p} P_{i k} Q_{k j}: P_{i k} \in A_{i k}, Q_{k j} \in B_{k j} \text { for } 1 \leq k \leq p\right\}
$$

For each $1 \leq i \leq m$, and each $j, 1 \leq j \leq n$.

Some of the solution of linear systems procedures are based an extension principle and arithmetic .On the other hand, Mehdi et al [1]. The interval matrix calculated with the account given in [2, 5,6,7].Consider

$$
\hat{A} \times \hat{X}=\hat{B}
$$

Matrix $\hat{A}$ in this equation represents the coefficient matrix , $\widehat{X}$ matrix is unknown and $\widehat{B}$ matrix right-hand side. System of linear equation the (1) can be rewritten as follows

$$
\left\{\begin{array}{c}
\left(\hat{a}_{11} \times \hat{x}_{1}\right)+\left(\hat{a}_{12} \times \hat{x}_{2}\right)+\cdots+\left(\hat{a}_{1 n} \times \hat{x}_{n}\right)=\hat{b}_{1} \\
\vdots \quad \vdots \\
\left(\hat{a}_{n 1} \times \hat{x}_{1}\right)+\left(\hat{a}_{n 2} \times \hat{x}_{2}\right)+\cdots+\left(\hat{a}_{n n} \times \hat{x}_{n}\right)=\hat{b}_{n}
\end{array}\right.
$$

\section{3:calculating interval inverse matrix}

In this section, some definition will be introduced in order to help in calculation inverse of an interval matrix. These are interval zero, interval one number and interval identity matrix.

\section{Definition (3-1): Identity matrix}

Ifleft-endpoint of an interval number is $1-\lambda$ and right-endpoint values are $1+\lambda$ where $0<\lambda<1$ this interval number us called interval one number and denoted by $\hat{1}=[1-\lambda, 1+\lambda]$. Also if left-endpoint of a interval number is $0-\lambda$ and right-endpoint values are $0+\lambda$ where $0<\lambda<1$ this interval number us called interval zero number and denoted by $\hat{0}=[0-\lambda, 0+\lambda]$. On this basis if the diagonal elements of a interval matrix are interval one number and the off-diagonal elements are interval zero numbers, then this interval matrix is called interval identity matrix and denoted by

$$
\hat{I}=\left[\begin{array}{ccc}
\hat{1} & \cdots & \hat{0} \\
\vdots & \ddots & \vdots \\
\hat{0} & \cdots & \hat{1}
\end{array}\right]
$$

In order to compute interval inverse matrix right-hand side vector equation system in (2) replays by interval identity matrix and can be rewritten as follows: 


$$
\left\{\begin{array}{c}
\left(\hat{a}_{11} \times \hat{x}_{11}\right)+\left(\hat{a}_{12} \times \hat{x}_{21}\right)+\cdots+\left(\hat{a}_{1 n} \times \hat{x}_{n 1}\right)=\hat{1} \\
\vdots \vdots \\
\left(\hat{a}_{n 1} \times \hat{x}_{1 n}\right)+\left(\hat{a}_{n 2} \times \hat{x}_{2 n}\right)+\cdots+\left(\hat{a}_{n n} \times \hat{x}_{n n}\right)=\hat{0}
\end{array}\right.
$$

The system which helps calculation the inverse of an interval matrix, to small examples will be given in the next section to illustrate the solution procedure.

\section{4: Implementation}

In this section two examples are given in order toillustrate the proposed method, Let $\hat{A}$ and $\hat{X}$ be two $2 \times 2$ interval matrix which is given as follows:

$$
\hat{A}=\left[\begin{array}{ll}
\hat{A}_{11} & \hat{A}_{12} \\
\hat{A}_{21} & \hat{A}_{22}
\end{array}\right] \quad, \quad \hat{X}=\left[\begin{array}{ll}
\hat{X}_{11} & \hat{X}_{12} \\
\hat{X}_{21} & \hat{X}_{22}
\end{array}\right]
$$

Where $\hat{X}$ is inverse matrix and both $\hat{A}_{i j}$ and $\hat{X}_{i j}, i, j=1,2$, are interval numbers. The system of equations that allows calculation the inverse of a real matrix is extended to interval case in order to calculate the inverse of an interval matrix using the so-called interval identity matrix.The symbolic form of the systems can be denoted as follows:

$$
\hat{A} \times \hat{X}=\hat{I}
$$

The expression given in (4) can be rewritten as follows:

$$
\left[\begin{array}{ll}
\hat{A}_{11} & \hat{A}_{12} \\
\hat{A}_{21} & \hat{A}_{22}
\end{array}\right] \times\left[\begin{array}{ll}
\hat{X}_{11} & \hat{X}_{12} \\
\hat{X}_{21} & \hat{X}_{22}
\end{array}\right]=\left[\begin{array}{ll}
\hat{1} & \hat{0} \\
\hat{0} & \hat{1}
\end{array}\right]
$$

Where $\hat{A}_{i j}=\left[\underline{A}_{i j}, \bar{A}_{i j}\right]$ and $\hat{X}_{i j}=\left[\underline{X}_{i j}, \bar{X}_{i j}\right], \mathrm{i}, \mathrm{j}=1,2$ and $\hat{1}=[1-\lambda, 1+\lambda], \hat{0}=[0-\lambda, 0+\lambda]$ and both are interval numbers. Suppose that two $2 \times 2$ interval matrix $\hat{A}$ and $\widehat{X}$, which consists interval numbers is as follows:

$$
\left[\begin{array}{ll}
{[1,2]} & {[3,4]} \\
{[5,6]} & {[7,8]}
\end{array}\right] \times\left[\begin{array}{ll}
{\left[\underline{X}_{11}, \bar{X}_{11}\right]} & {\left[\underline{X}_{12}, \bar{X}_{12}\right]} \\
{\left[\underline{X}_{21}, \bar{X}_{21}\right]} & {\left[\underline{X}_{22}, \bar{X}_{22}\right]}
\end{array}\right]=\left[\begin{array}{ll}
{[1-\lambda, 1+\lambda]} & {[0-\lambda, 0+\lambda]} \\
{[0-\lambda, 0+\lambda]} & {[1-\lambda, 1+\lambda]}
\end{array}\right]
$$

Assuming the values $\hat{A}_{i j}>0$, then matrix multiplication can be written in the form interval linear equation system as follows:

$$
\begin{array}{r}
{[1,2]\left[\underline{X}_{11}, \bar{X}_{11}\right]+[3,4]\left[\underline{X}_{21}, \bar{X}_{21}\right]=[1-\lambda, 1+\lambda]} \\
{[1,2]\left[\underline{X}_{12}, \bar{X}_{12}\right]+[3,4]\left[\underline{X}_{22}, \bar{X}_{22}\right]=[0-\lambda, 0+\lambda]} \\
{[5,6]\left[\underline{X}_{12}, \bar{X}_{12}\right]+[7,8]\left[\underline{X}_{21}, \bar{X}_{21}\right]=[0-\lambda, 0+\lambda]} \\
{[5,6]\left[\underline{X}_{12}, \bar{X}_{12}\right]+[7,8]\left[\underline{X}_{22}, \bar{X}_{22}\right]=[1-\lambda, 1+\lambda]}
\end{array}
$$

The equation system (6) can be written as follows: 


$$
\begin{gathered}
{\left[2 \bar{X}_{11}, \underline{X}_{11}\right]+\left[4 \bar{X}_{21}, 3 \underline{X}_{21}\right]=[1-\lambda, 1+\lambda]} \\
{\left[2 \bar{X}_{12}, \underline{X}_{12}\right]+\left[4 \bar{X}_{22}, 3 \underline{X}_{22}\right]=[0-\lambda, 0+\lambda]} \\
{\left[6 \bar{X}_{11}, 5 \underline{X}_{11}\right]+\left[8 \bar{X}_{21}, 7 \underline{X}_{21}\right]=[0-\lambda, 0+\lambda]} \\
{\left[6 \bar{X}_{12}, 5 \underline{X}_{12}\right]+\left[8 \bar{X}_{22}, 7 \underline{X}_{22}\right]=[1-\lambda, 1+\lambda]}
\end{gathered}
$$

The equation systems in (7) is split in to two separate equation system where (I ) system are called values left-endpoints and (II) equation system are called value right-endpoint

I )

$$
\left\{\begin{array}{rlrl}
2 \bar{X}_{11}+4 \bar{X}_{21}=1-\lambda & & 2 \bar{X}_{12}+4 \bar{X}_{22}=0-\lambda \\
6 \bar{X}_{11}+8 \bar{X}_{21}=0-\lambda & 6 \bar{X}_{12}+8 \bar{X}_{22}=1-\lambda
\end{array}\right.
$$

II ) $\left\{\begin{array}{rl}\underline{X}_{11}+3 \underline{X}_{21}=1+\lambda \\ 5 \underline{X}_{11}+7 \underline{X}_{21}=0+\lambda\end{array} \quad, \quad \begin{array}{ll}\underline{X}_{12}+3 \underline{X}_{22}=0+\lambda \\ 5 \underline{X}_{12}+7 \underline{X}_{22}=1+\lambda\end{array}\right.$

Then, the values of the variables (I), (II) equations systems are:

$$
\begin{array}{lrl}
\underline{X}_{11}=-1.1 & \underline{X}_{12}=0.125 \\
\underline{X}_{21}=0.87 & \underline{X}_{22}=0.125 \\
\bar{X}_{11}=-0.75 & \bar{X}_{12}=0.75 \\
\bar{X}_{21}=0.50 & \bar{X}_{22}=-0.50
\end{array}
$$

Thus, the inverse matrix of the interval matrix can be calculated as a solution of the equation system given in (7). As stated before, the values $0<\lambda<1$. There for, the $\hat{X}_{i j} \mathrm{i}, \mathrm{j}=1,2$ values should be examined by changing $\lambda$ between $(0,1)$ open interval. These values are summarized in Table (1)

Table1(when parameters increasing in the same direction)

\begin{tabular}{clllllllll}
\hline $\boldsymbol{\lambda}, \boldsymbol{\alpha}$ &. $\mathbf{1}$ & $\mathbf{. 2}$ & $\mathbf{. 3}$ & $\mathbf{. 4}$ & $\mathbf{. 5}$ & $\mathbf{. 6}$ & $\mathbf{. 7}$ & $\mathbf{. 8}$ & $\mathbf{. 9 9}$ \\
\hline$\overline{\boldsymbol{X}_{\mathbf{1 1}}}$ & -.095 & -.90 & -.85 & -.80 & -.75 & -.70 & -.65 & -.60 & -.50 \\
$\overline{\boldsymbol{X}_{\mathbf{1 2}}}$ & .55 & .60 & .65 & .70 & .75 & .80 & .85 & .90 & .99 \\
$\overline{\boldsymbol{X}_{\mathbf{2 1}}}$ & .70 & .65 & .60 & .55 & .50 & .45 & .40 & .35 & .25 \\
$\overline{\boldsymbol{X}_{\mathbf{2 2}}}$ & -.30 & -.35 & -.40 & -.45 & -.50 & -.55 & -.60 & -.65 & -.74 \\
\hline
\end{tabular}

Table 1 show that when $\lambda$ is taken as fix values without considering the effects on $\hat{X}_{i j}$ values of the elements in the inverse matrix, the resulting interval inverse matrix could have wider interval. There for the goal is that $\lambda$ is being tried to be kept as small as possible. When Table 1 is closely examined we see that, all values right-endpoint the coefficients $\lambda$ can increase or decrease.

For example let say $\bar{x}_{11}$ when $\lambda$ increases for 0.10 to 0.99 it takes values from -0.095 to -0.50 , therefore, choosing left or right-endpoint parameters is merely a decision making problem which is dependent upon the researcher. 
We examined how the $\bar{X}_{i j}$ values have the best right-endpoint of the interval inverse matrix for our example. According to these obtained results the best right-endpoint values are determined by the decision maker. Where the best values can be fixed such $(0.50)$.Since the values more than 0.50 is expected the more open interval and the values less than 0.50 artifacts are more limited rang

Then let say $\lambda=0.50$ the inverse of $\hat{A}$ can be written as follows:

$$
A^{-1}=\left[\begin{array}{cc}
{[-1.1,-0.75]} & {[.125,0.75]} \\
{[0.50,0.87]} & {[-0.50,0.125]}
\end{array}\right]
$$

Second illustration uses negative interval matrix. In other words, $\hat{A}_{i j}<0$. Let B be a $2 \times 2$ interval matrix where $\lambda=.50$

$$
B=\left[\begin{array}{ll}
{[-2,-1]} & {[-4,-3]} \\
{[-6,-5]} & {[-8,-7]}
\end{array}\right]
$$

Then the some calculation presented in the first example the resulting interval inverse matrix is given as follows:

$$
B^{-1}=\left[\begin{array}{cc}
{[0.62,1.25]} & {[-0.62,-0.125]} \\
{[-1,-.37]} & {[0,0.37]}
\end{array}\right]
$$

Interval matrices has assessed in all cases except [8].

So, the both matrix $A^{-1}, B^{-1}$ are inverses matrix $\mathrm{A}, \mathrm{B}$ is weak.

\section{5: Conclusion}

In this paper, a method is proposed in order to calculation the inverse of an interval matrix the equation as it is $\hat{A} \times \hat{X}=\widehat{B}$ it can calculated using matrix inversion. Thus by definition zero interval number, one interval number we could define an interval identity matrix.Assuming $\hat{A}$ is an interval coefficient matrix, $\hat{X}$ is unknown interval matrix and $\widehat{B}$ is an interval identity matrix we solved linear equation systems. One of the things it should be noted that, the solution of the constructed interval linear system is parameter depended unless one specifies the right-endpoint values of interval identity matrix in advance. When the system is solved in the parametric form the best solution is chosen among them by decision maker. The other hand if one is not interested in multiple solution right-end point can be fixed such as 0.50 . So after finding the $2 \times 2$ inverse matrix, we can say: If all intervals are positive, we can conclude that the right-end point of the interval of the second row is identical but finding the $\bar{X}_{11}$ signs of the change. The left-right end points entries the second row is interchange. In no case $\hat{A}_{i j}<0$ the same thing happens of the first row. If $\bar{A}_{i i}=0$ in that a $2 \times 2$ interval matrix left and rights end points of the diameter range is placed.

\section{References}

[1] Murat AlperBasaran, Amethod for calculating interval inverse matrix, Applied soft computing 12 (2012) 1810-1813 
[2] Sh. Abolmasoumi, M. Alavi, A method for calculating interval linear system, Journal of mathematics and computer science 8 (2014), 193-204.

[3] M. Dehghan, H. Hashemi, M. Ghatee, computational methods for solving fully fuzzy linear systems, Applied Mathematics and computation 179(2006) 328-343

[4] D. Dubois, H.Prade, Fuzzy sets and systems: Theory and Application, Academic press, new York, 1980

[5] E. Hansen, Interval arithmetic in matrix computation, Part I, SIAM Journal on Numerical Analysis 2 (1965) 308-320

[6] E. Hansen, Interval arithmetic in matrix computation , Part II, SIAM Journal on Numerical Analysis 4 (1967) 1-9

[7] V. Kreinovich, A.V. Lakeyev, J.Rohn, P.T.Kahl, Computational Complexity and Feasibility, Of Numerical Method in Engineering 42 (1998) 829-846

[8] Ramon E. Moor, R Baker Kear foot, Michael .j.cloud, Introduction to interval Analysis 3600market street, 6th floor, Philadelphia , PA, 19104-2688 USA.p.cm. 